\title{
Search of MeV-GeV Counterparts of TeV Sources
}

\author{
Carlotta Pittori ${ }^{* \dagger}$ \\ INAF-OAR/ASDC, Roma, Italy \\ E-mail: carlotta.pittori@asdc.asi.it
}

\section{A. Rappoldi}

INFN-Pavia, Pavia, Italy

\section{F. Lucarelli}

INAF-OAR/ASDC, Roma, Italy

P.W. Cattaneo

INFN-Pavia, Pavia, Italy

\section{F. Longo}

INAF-OAT and INFN-Trieste, Trieste, Italy

\section{F. Verrecchia}

INAF-OAR/ASDC, Roma, Italy

\begin{abstract}
We present the results of a systematic search for gamma-ray emission from known and unidentified $\mathrm{TeV}$ sources, using the data collected by the AGILE satellite in observing pointing mode in the energy range above $100 \mathrm{MeV}$. We found that $\sim 34 \%$ of the analysed $\mathrm{TeV}$ sources sample, extracted from the online TeVCat catalog, show a significant excess in the AGILE data collected during the pointing observation period, from July 9, 2007 to October 18, 2009. We plan to extend this analysis also to the AGILE data collected from November 2009 on, obtained by observing each day a large portion of the sky in spinning mode.
\end{abstract}

Frontier Research in Astrophysics - II

23-28 May 2016

Mondello (Palermo), Italy

* Speaker.

${ }^{\dagger}$ on behalf of the AGILE Collaboration. 


\section{Introduction}

After more than a decade of operation, the current generation of ground-based high-sensitivity imaging atmospheric Cherenkov telescopes (H.E.S.S., MAGIC, and VERITAS) have now detected more than 150 VHE gamma-ray sources. These comprise many different source classes: pulsars and their nebulae, supernova remnants, X-rays binary systems, and active galactic nuclei. Moreover a significant fraction of them $(\sim 20 \%)$ still remains unidentified (UNID), and multi-wavelength observations are needed both to identify the possible counterparts of the UNID, as well as to understand the emission mechanisms of the $\mathrm{TeV} \gamma$-rays.

Previous studies on the positional and spectral connection of $\mathrm{GeV}$ to $\mathrm{TeV} \gamma$-ray sources performed on EGRET and Fermi-LAT satellite data indicate in particular that a single spectral component seems often unable to describe the $\mathrm{MeV}$ to $\mathrm{TeV}$ spectra of many coincident $\mathrm{GeV} / \mathrm{TeV}$ sources,

This search is particularly relevant since the two adjacent energy ranges may probe different regions of the source spectra, so we performed a systematic analysis on a sample of the known $\mathrm{TeV}$ sources with the purpose of detecting $\gamma$-ray emission associated with the $\mathrm{TeV}$ sources using the AGILE MeV-GeV $\gamma$-ray satellite data [1].

\section{The TeV sources input sample}

The online TeVCat catalog [2] is continuously updated with new sources detected by $\mathrm{TeV}$ experiments, and at the time of the analysis (June 2015) contained a total of $183 \mathrm{TeV}$ sources. The analysis was performed on a subset of $152 \mathrm{TeV}$ sources, both galactic and extragalactic, consisting of 120 sources of the 'default catalog', plus 32 'newly announced' sources. Sources flagged as 'other sources', 'sources candidates', and 'extended regions' already including a compact TeV source were excluded in the definition of the input sample.

Each sky position and extension of the TeV sources in the selected TeVCat sample has been carefully reviewed using published data [3]. A new interactive web page of the catalog of $\mathrm{TeV}$ sources, including this coordinate revision and giving public access to light curves and spectra, is now available at the ASI Science Data Center (ASDC) ${ }^{1}$. The error region on each TeV source position has been calculated by quadratically summing the statistical uncertainties on the position coordinates and the systematic uncertainties on the instrument pointing, when available.

\section{The AGILE data set and the analysis procedure}

AGILE [4] is an Italian Space Agency (ASI) small scientific mission for high-energy astrophysics launched on April 23, 2007 in an equatorial orbit with initial altitude of about $550 \mathrm{~km}$. The analysis has been performed using the data collected by the main AGILE instrument, the GammaRay Imaging Detector (GRID), sensitive in the energy range $30 \mathrm{MeV}-50 \mathrm{GeV}$. The AGILE-GRID consists of a pair conversion silicon-tungsten tracker, a caesium iodide mini-calorimeter, and an anticoincidence system. The payload is a small cube of $\sim 60 \mathrm{~cm}$ size, which achieves an effective area

\footnotetext{
${ }^{1}$ http://www.asdc.asi.it/tgevcat/
} 
of approximately $500 \mathrm{~cm}^{2}$ at several hundreds $\mathrm{MeV}$, an angular resolution (at $68 \%$ containment radius) of about $4.3^{\circ}$ at $100 \mathrm{MeV}$, decreasing below $1^{\circ}$ for energies above $1 \mathrm{GeV}$, an unprecedentedly large field of view (FOV) of about $\sim 2.5 \mathrm{sr}$.

The analysed AGILE data set covers the period of about two years, from July 9, 2007 (beginning of the science verification phase) to October 18, 2009, during which the satellite was operated in 'pointing observing mode'. During this period, following the main scientific program of the AGILE mission, the satellite observation strategy was mainly focused on the Galactic plane, and was not optimal for detecting extragalactic $\mathrm{TeV}$ sources.

An iterative automated procedure has been developed to analyse the entire pointing AGILE data set searching for possible $\gamma$-ray excesses correlated to the $\mathrm{TeV}$ sources.

The first part of the procedure is the creation of the AGILE maps of counts, exposure and diffuse $\gamma$-ray background using updated calibrations, and centered at the input $\mathrm{TeV}$ source positions. Maps were generated for energies E $>100 \mathrm{MeV}$, including all events collected up to $60^{\circ}$ off-axis. The South Atlantic Anomaly data were excluded and, to eliminate the Earth albedo contamination, events with reconstructed directions with respect to the satellite-Earth vector that were smaller than $85^{\circ}$ were also rejected.

The second part of the analysis consists in verifying if, around the input $\mathrm{TeV}$ position, it is possible to detect the presence of a significant $\gamma$-ray source by means of a multi-source maximum likelihood estimator (MLE) algorithm and, if so, to try to locate the best position of the $\gamma$-ray excess. An iterative MLE analysis was performed taking into account all the already known $\gamma$-ray sources detected by AGILE at the time of the analysis, assumed to be point-like with simple powerlaw spectra. In particular, we considered all the published AGILE sources, consisting of a set of 65 sources obtained by combining the 1AGL/1AGLR catalogs [5, 6], and two AGL sources in the Carina region (see also Table 2 and Table 3 of 1AGLR paper), plus one additional AGL source from a detailed analysis of the Cygnus region [7].

The significance of a source detection is evaluated by the square root of the test statistic TS, which for a large enough number of counts $(N \gtrsim 20)$ is expected to behave as $\chi_{1}^{2}$ in the null hypothesis. An AGILE 'detection' was in general defined by the condition

$$
\sqrt{(T S)_{4}} \geq 4
$$

which corresponds to a statistical significance of about $4 \sigma$ at the input $\mathrm{TeV}$ source position. The search for optimised positions of the $\gamma$-ray excess gives results that are considered reliable when the MLE analysis converges well within the allowed searching distance from the input position, and the significance of the detection is increased.

\section{Results}

For significant sources according to the criteria described above, the calculated flux value and the corresponding error was given, while for the sources not satisfying the detection requirement, the estimated 95\% CL upper limit was reported.

In total, $52 \mathrm{TeV}$ sources show a significant excess in the AGILE data that covers the pointed observation period, corresponding to $34 \%$ of the original sample. The Aitoff map in Galactic coordinates of all the detections is shown in Fig. 1. In this analisys 26 new AGILE sources are 
found with respect to the AGILE reference catalogs , 15 of which are galactic, 7 are extragalactic and 4 are unidentified. Eight sources detected by AGILE in this analysis have no Fermi 3FGL official TeV association, two of which do not represent new gamma-ray detections, since they have counterparts in the already published AGILE catalogs. The majority of the AGILE detected sources are galactic. This might be a bias due the higher exposure of the Galactic plane during the pointing observation period.

The spatial association of a TeV source with an AGILE source may be due to chance. The probability of a serendipitous association has been estimated by evaluating the total sky coverage, and it is found to be 0.82 around the Galactic plane, whereas it is 0.08 for the other sky regions. Therefore the chance coincidence should be $\mathrm{O}(1)$ over the whole sky.

All the results can be found at the new AGILE interactive webpage dedicated to the AGILEGRID TeV counterparts catalog: http://www.asdc.asi.it/agiletevcat/, including images of the AGILE GRID intensity maps available through the "ASDC Data Explorer" in the 'AGILE GRID Data Products' tab.

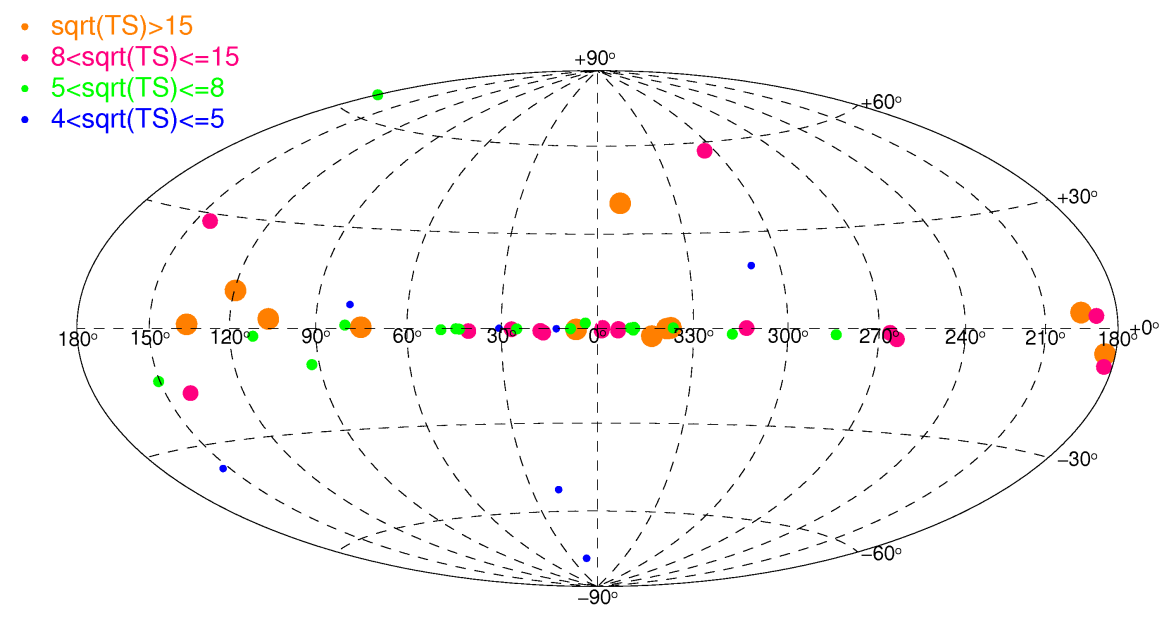

Figure 1: Aitoff map in Galactic coordinates of all the detections according to the criteria specified in the text, corresponding to $\mathrm{TeV}$ sources that show a significant excess in the AGILE data. See also: http://www.asdc.asi.it/agiletevcat/

\section{References}

[1] Rappoldi, A. et al., A.\&A. 587, (2016) A93

[2] Wakely, S. and Horan, D., http://tevcat.uchicago.edu

[3] Carosi, A. et al., Proc. SPIE 9913 (2016), doi:10.1117/12.2231654

[4] Tavani, M. et al., A.\&A. 502, (2009) 995

[5] Pittori, C. et al., A.\&A. 506, (2009) 1563

[6] Verrecchia, F. et al., A.\&A. 558, (2013) A137

[7] Bulgarelli, A. et al. A.\&A. 538, (2012), A63 